\title{
Value of sentinel node status as a prognostic factor in melanoma: prospective observational study
}

Stephen Kettlewell, Colin Moyes, Caroline Bray, David Soutar, Alan MacKay, Dominique Byrne, Taimur Shoaib, Barun Majumder, Rona MacKie

\begin{abstract}
Objective To establish the prognostic value of knowledge of sentinel node status in melanoma.

Design Single centre prospective observational study, with sentinel nodes identified by lymphoscintigraphy, $\gamma$ probe, and intraoperative blue dye and examined by both conventional histopathology and immunopathology.

Setting Specialist surgical service in west of Scotland.

Participants 482 patients with melanoma who consented to sentinel node biopsy in 1996-2003.

Main outcome measure Time to recurrence of or death from melanoma.

Results Of 472 patients who consented to sentinel node biopsy and in whom at least one sentinel node was identified, 367

$(78 \%)$ had no tumour in the sentinel node. At mean follow-up of 42 months, 299 (82\%) of this group were alive and free from disease, 24 were alive with melanoma recurrence, and 31 had died of melanoma. Of 105 patients with a positive sentinel node biopsy, 44 (42\%) were alive and disease free, 12 were alive with recurrence, and 46 had died of melanoma. The survival difference between patients who were negative and those who were positive for tumour in the sentinel node was highly significant at all thickness levels over $1.0 \mathrm{~mm}(\mathrm{P}<0.001)$. Multivariate analysis showed that sentinel node status was independent of tumour thickness and ulceration. 71/105 (68\%) patients with a positive sentinel node had a negative completion lymphadenectomy, and 44/71 (62\%) were alive and disease free at follow-up; 34 patients with a positive sentinel node had further nodes involved, and only $4(12 \%)$ were disease free $(\mathrm{P}<0.001) .16$ patients $(13$ sentinel node biopsy positive; 3 negative) died of other causes.

Conclusion Sentinel node status is a highly significant predictor of prognosis in melanoma and should be considered in adjuvant studies. However, it should not be regarded as a standard of care until mature data from ongoing randomised trials are available.
\end{abstract}

\section{Introduction}

The technique of sentinel node biopsy (SNB) was first introduced for genital cancer, ${ }^{1}$ and, in the past decade, it has been widely used both for patients with melanoma and for those with breast cancer. ${ }^{2-10}$ In breast cancer, SNB is becoming a standard method of nodal sampling, and the presence or absence of tumour cells in the node determines the treatment pathway after surgery. ${ }^{11}$ For melanoma, the lack of proved effective nonsurgical adjuvant treatment precludes this approach. A multicentre randomised trial (MSLT1) is in progress with the aim of determining if patients with melanoma who have a positive SNB and proceed immediately to full node dissection have a superior disease-free survival or overall survival compared with patients who have node dissection only when nodes draining the site of the primary melanoma are clinically palpable. Definitive results are awaited. ${ }^{12}$

This study started before MSLT1. We aimed to gain clinical experience of the technique of SNB in a single centre and determine whether sentinel node status adds prognostic information to that gained from measuring tumour thickness.

\section{Methods}

We identified 482 patients, who gave written consent to take part in the study. All patients had an appropriate wide excision of their primary melanoma; if the primary lesion was $1 \mathrm{~mm}$ or thicker, we invited them to participate in the study; all agreed to participate. We did all SNBs within eight weeks of surgery for the primary tumour, and no patient had clinical or biochemical evidence of spread beyond the primary tumour site. The day before $\mathrm{SNB}$, patients had lymphoscintigraphy with ${ }^{99 \mathrm{~m}} \mathrm{Tc}$ nanocolloid to identify the appropriate draining nodal basin to sample. All patients had general anaesthesia for the SNB. We injected 0.1-1.5 $\mathrm{ml}$ of patent blue $\mathrm{V}$ dye intradermally adjacent to the scar at the primary melanoma site. We then exposed the relevant nodal basin and used the tracking of blue dye to identify the sentinel node. We confirmed this with a hand held collimated $\gamma$ radiation detection probe (Neoprobe Corporation), which we also used after excision of the sentinel node(s) to confirm completeness of excision.

The pathologist who received the nodes divided them along the long axis, cut up to $5 \times 5 \mu \mathrm{m}$ sections from each face, and stained them both with routine haematoxylin and eosin and with antibodies to $\mathrm{S} 100$ protein and melan A/Mart. If melanoma cells were identified on these sections, no further sections were cut. If these sections were negative, the pathologist cut up to 20-30 further sections and stained them alternately with haematoxylin and eosin or melanoma antibodies. We recorded the volume and site within the node of melanoma cells. All patients with one or more positive SNBs proceeded to completion lymphadenectomy within four weeks.

We followed up patients at intervals of three months after SNB with clinical, haematological, and biochemical examination, and chest radiography. We ordered computed tomography or magnetic resonance imaging only if clinical signs suggested recurrence of melanoma. 
Table 1 Number of patients with positive sentinel node biopsy (SNB) by thickness, and current status according to SNB findings. Values are numbers (percentages)

\begin{tabular}{|c|c|c|c|c|c|c|c|c|c|c|c|c|}
\hline \multirow{3}{*}{$\begin{array}{l}\text { Thickness of } \\
\text { primary } \\
\text { melanoma (mm) }\end{array}$} & \multicolumn{2}{|c|}{ All cases* } & \multicolumn{5}{|c|}{ SNB negative† } & \multicolumn{5}{|c|}{ SNB positive† } \\
\hline & \multirow[b]{2}{*}{ All } & \multirow[b]{2}{*}{$\begin{array}{c}\text { SNB } \\
\text { positive }\end{array}$} & \multirow[b]{2}{*}{ All } & \multicolumn{2}{|c|}{ Alive } & \multicolumn{2}{|c|}{ Died } & \multirow[b]{2}{*}{ All } & \multicolumn{2}{|c|}{ Alive } & \multicolumn{2}{|c|}{ Died } \\
\hline & & & & $\begin{array}{l}\text { Disease } \\
\text { free }\end{array}$ & $\begin{array}{c}\text { With } \\
\text { disease }\end{array}$ & Of melanoma & $\begin{array}{l}\text { Other } \\
\text { cause }\end{array}$ & & $\begin{array}{c}\text { Disease } \\
\text { free }\end{array}$ & $\begin{array}{c}\text { With } \\
\text { disease }\end{array}$ & Of melanoma & $\begin{array}{l}\text { Other } \\
\text { cause }\end{array}$ \\
\hline $0-0.9$ & 5 & 0 & 5 & 5 & 0 & 0 & 0 & 0 & 0 & 0 & 0 & 0 \\
\hline $1-1.9$ & 163 & $19(12)$ & 144 & $136(94)$ & 4 & 2 & 2 & 19 & $14(73)$ & 1 & 4 & 0 \\
\hline $2-2.9$ & 101 & $19(19)$ & 82 & $70(85)$ & 5 & 5 & 2 & 19 & $6(32)$ & 3 & 9 & 1 \\
\hline $3-3.9$ & 60 & $18(30)$ & 42 & $35(83)$ & 2 & 5 & 0 & 18 & $10(55)$ & 1 & 7 & 0 \\
\hline$\geq 4$ & 143 & 49 (34) & 94 & $53(56)$ & 13 & 19 & 9 & 49 & 14 (29) & 7 & 26 & 2 \\
\hline$\overline{\text { All }}$ & 472 & 105 (22) & 367 & $299(82)$ & 24 & 31 & 13 & 105 & $44(42)$ & 12 & 46 & 3 \\
\hline
\end{tabular}

*Percentage SNB positive increases significantly with increasing thickness range $\left(P<0.001, \chi^{2}\right.$ test for trend).

†For all patients, and for each thickness category, the difference in disease-free survival between SNB positive and SNB negative patients is highly significant $(P<0.001)$.

We recorded the times to recurrence and to death. We used the Cox model for multivariate analysis to evaluate the prognostic significance of sentinel node status for survival as compared with thickness of primary tumour, ulceration of the primary lesion, and other postulated prognostic features. We used Kaplan-Meier survival curves and the log rank test to investigate any differences in survival between SNB positive and SNB negative patients in all thickness categories, and also in the separate thickness categories 1-1.9 mm, 2-2.9 mm, 3-3.9 mm, and $4 \mathrm{~mm}$ and thicker.

\section{Results}

We attempted $482 \mathrm{SNB}$ procedures and identified one or more sentinel nodes in all but 10 cases. The median number of sentinel nodes identified was two (range one to five). Among the patients with nodes identified, 207 were men and 265 were women, with a median age at diagnosis of melanoma of 54 (range 17-89) years. Most primary lesions were on the lower limb (225 cases, $48 \%)$ with $88(19 \%)$ on the upper limb, $108(23 \%)$ on the trunk, and $51(11 \%)$ on the head or neck. Most primary lesions $(253 / 472,54 \%)$ were superficial spreading melanomas; $125(27 \%)$ were nodular primary tumours, $31(7 \%)$ were acral melanomas, $10(2 \%)$ were lentigo maligna melanomas, and 53 were unclassifiable. Two patients had an allergic reaction to blue dye, but no other serious toxicity occurred.

Table 1 shows the number and percentage of positive sentinel node biopsies, both in total and also divided into primary tumour thickness categories. The right hand side of the table gives details of the status of all patients at follow-up, divided into patients with a negative sentinel node biopsy and those with a positive sentinel node biopsy. One hundred and five patients (22\%) had one or more positive sentinel nodes, and $34(32 \%)$ of these had further nodes containing melanoma identified at completion lymphadenectomy.

Five patients with primary melanomas $<1.0 \mathrm{~mm}$ had SNB for no obvious reason such as ulceration or a Clark level 4 primary melanoma. We excluded these from the statistical analysis. All five had negative SNBs and were alive and disease free at follow-up.

The incidence of positive SNBs rose from $12 \%$ to $19 \%$ to $30 \%$ to $34 \%$ with increasing thickness category, a statistically significant trend $\left(\mathrm{P}<0.001, \chi^{2}\right.$ test for trend). Follow-up to a maximum of 130 months (mean 42 months) found that for all 367 SNB negative patients recurrence-free survival was $82 \%$, whereas recurrence-free survival for all $105 \mathrm{SNB}$ positive patients was $42 \%$, a highly significant difference $(\mathrm{P}<0.001)$.

Twenty four of $367 \mathrm{SNB}$ negative patients were alive with recurrent melanoma at follow-up, and 31 SNB negative patients had died of melanoma. Thus 55/367 (15\%) of SNB negative patients had disease progression at follow-up. For comparison, $12 \mathrm{SNB}$ positive patients were alive with recurrence and $46 \mathrm{had}$ died of melanoma, a combined recurrence rate of 58/105 (55\%). More detailed analysis showed that $71 / 105$ (67\%) SNB positive patients had a negative completion lymphadenectomy, and 34 $(32 \%)$ had additional nodes containing melanoma. Forty four of the $71(62 \%)$ SNB positive completion, lymphadenectomy negative patients were alive and recurrence free, 4 were alive with recurrent melanoma, 19 had died of melanoma. and 3 had died of other causes. In contrast, only 4/34 (12\%) SNB positive, completion lymphadenectomy positive patients were alive and recurrence free, 3 were alive with recurrence, and 27 had died of melanoma. This difference in survival was highly significant $(\mathrm{P}<0.001)$.

Slides from the sentinel node biopsies of the 55 patients reported as SNB negative but who had disease progression at follow-up have been independently reviewed and do not suggest that the original report of a negative SNB was incorrect. The first site of recurrence in the $31 \mathrm{SNB}$ negative patients who died of melanoma was nodal in $12(39 \%)$, distant in $14(45 \%)$, and in transit in $5(16 \%)$; for the $39 \mathrm{SNB}$ positive patients the site was nodal in $4(10 \%)$, distant in $30(77 \%)$, and in transit in $4(10 \%)$.

Table 2 shows the multivariate analysis for the group. The most powerful prognostic factor for survival was the sentinel node status; ulceration and tumour thickness were independent prognostic factors. The hazard ratio for a positive sentinel node in a Cox model including only primary tumour thickness and ulceration as additional variables was 4.15 (95\% confidence interval 2.82 to 6.11 ). Clark levels, age at melanoma diagnosis, sex, and body site of primary tumour did not add any additional prognostic information. When we analysed the SNB negative and SNB positive groups separately, tumour thickness was retained in the model as significant for both groups but ulceration lost independent significance for the SNB positive group.

The figure shows Kaplan-Meier survival curves for the entire group and for patients with tumours in the $1-1.9 \mathrm{~mm}, 2-2.9 \mathrm{~mm}$, 3-3.9 mm, and $\geq 4 \mathrm{~mm}$ thickness groups. They show statistically significant survival differences in all thickness categories between SNB positive and SNB negative patients $(\mathrm{P}<0.001)$. These curves clearly show that sentinel node status in all thickness categories adds additional significant prognostic information to that already obtained from measurements of primary tumour thickness. 
Table 2 Cox's proportional hazard model comparing sentinel node status with other postulated prognostic factors as independent influences on survival

\begin{tabular}{|c|c|c|c|}
\hline Factors & No* & Hazard ratio $(95 \%$ Cl) & $P$ value \\
\hline \multicolumn{4}{|c|}{ Sentinel node biopsy } \\
\hline Positive & 99 & 4.40 (2.91 to 6.64$)$ & $<0.001$ \\
\hline Negative & 352 & 1 & \\
\hline \multicolumn{4}{|l|}{ Ulceration } \\
\hline Yes & 124 & 1.80 (1.20 to 2.70$)$ & 0.005 \\
\hline Other & 327 & 1 & \\
\hline \multicolumn{4}{|c|}{ Tumour thickness (mm) } \\
\hline $1-1.99$ & 159 & 1 & \\
\hline $2-2.99$ & 96 & 2.41 (1.11 to 5.22) & 0.026 \\
\hline $3-3.99$ & 58 & 1.70 (0.71 to 4.11$)$ & 0.236 \\
\hline$\geq 4$ & 138 & 4.36 (2.13 to 8.91$)$ & $<0.001$ \\
\hline \multicolumn{4}{|l|}{ Clark level } \\
\hline 2 & 20 & 0.50 (0.12 to 2.06$)$ & 0.335 \\
\hline 3 & 75 & 0.56 (0.25 to 1.24$)$ & 0.153 \\
\hline 4 & 301 & 1 & \\
\hline 5 & 44 & 1.08 (0.60 to 1.93$)$ & 0.801 \\
\hline Not assessable & 11 & 0.47 (0.14 to 1.59$)$ & 0.225 \\
\hline \multicolumn{4}{|l|}{ Age (years) } \\
\hline$<40$ & 93 & 0.46 (0.23 to 0.92$)$ & 0.028 \\
\hline 40 to 59 & 174 & 1 & \\
\hline$\geq 60$ & 184 & 0.76 (0.48 to 1.21$)$ & 0.253 \\
\hline \multicolumn{4}{|l|}{ Sex } \\
\hline Male & 201 & 1.08 (0.69 to 1.69$)$ & 0.742 \\
\hline Female & 250 & 1 & \\
\hline \multicolumn{4}{|l|}{ Body site } \\
\hline Limbs & 298 & 1 & \\
\hline Head and neck & 48 & 1.14 (0.53 to 2.45$)$ & 0.731 \\
\hline Trunk & 105 & 1.51 (0.90 to 2.53$)$ & 0.118 \\
\hline
\end{tabular}

*Number of patients included in analysis=451 (number of cases with complete data for all variables).

\section{Discussion}

Over the past decade, the use of the sentinel node biopsy technique has become commonplace in many melanoma treatment centres in Europe, North America, and Australia despite a lack of evidence of any survival advantage. The randomised trial MSLT1 is designed to resolve this issue, and results in a refereed paper are eagerly awaited. The most up to date report is from the American Society of Clinical Oncology's website, which gives results as at April 2005. This reports that for patients with tumours 1.2-3.5 mm thick, the "wide excision and wait" arm of the trial has a five year overall survival of $86 \%$, compared with a five year overall survival of $87 \%$ for the wide excision and SNB group (no significant difference). ${ }^{12}$

Before 1990 the most widely practised approach to management of lymph nodes in patients with primary melanoma in Europe was to delay nodal sampling until clinical evidence suggested nodal involvement. A fine needle aspirate or node biopsy was then done, and a completion lymphadenectomy was done if either showed melanoma cells. The introduction of SNB in Europe has thus added an additional surgical procedure involving general anaesthesia shortly after excision of the primary melanoma. Our results, and those of others, all indicate that only around $20 \%$ of these biopsies reveal the presence of melanoma cells. $^{4512}$

In contrast, in North America and in some Australian centres, common practice before the availability of SNB was to do an elective node dissection for patients with melanomas thicker than $1 \mathrm{~mm}$ at the time of primary surgery. Thus, in these countries, the current use of sentinel node biopsies saves SNB negative patients an unnecessary elective node dissection with the attendant morbidity of this procedure, such as lymphooedema that can be both troublesome and persistent.

\section{Recommendations}

We consider that SNB should not be regarded as the standard of care in European centres and in other countries where a conservative approach to lymphadenectomy for AJCC stages 1 and 2 melanoma has been the norm until final results from the MSLT1 randomised trial are available for consideration. At that
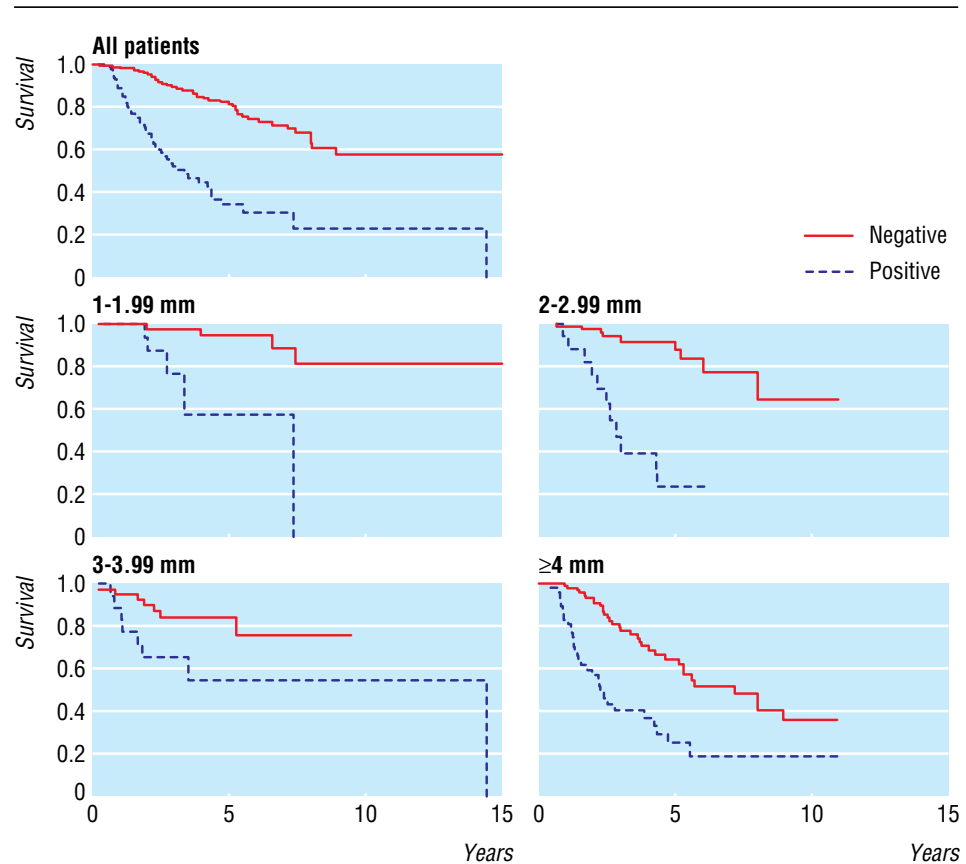

Kaplan-Meier survival curves for patients with positive and negative sentinel node biopsies for all tumour thicknesses $(P<0.001)$ and for patients in categories of $1-1.99$ $\mathrm{mm}(\mathrm{P}<0.001), 2-2.99 \mathrm{~mm}(\mathrm{P}<0.001), 3-3.99 \mathrm{~mm}(\mathrm{P}<0.05)$, and $\geq 4 \mathrm{~mm}(\mathrm{P}<0.001)$ tumour thickness 
point, consideration of which patient group is most likely to benefit may be needed.

\section{Need for trials of adjuvant treatment}

Our single centre European study reviews a large series of patients who have had $\mathrm{SNB}$ and adequate follow-up to determine the relation between tumour thickness, SNB status, and survival. These data clearly show that SNB status adds important prognostic information to that provided by tumour thickness at the time of diagnosis in all thickness groupings. This is maintained for patients with primary melanomas thicker than $4 \mathrm{~mm}$, and this group should not be regarded as having uniformly fatal disease. We have also shown the very poor prognosis for patients with primary melanomas of any thickness who have additional non-sentinel nodes involved at full node dissection, strongly implying that these patients already have disseminated disease. A very strong case exists for a trial of adjuvant treatment directed specifically at patients with SNB positive, completion lymphadenectomy positive melanoma. Our data, unlike many other trials of adjuvant treatment in melanoma, suggest that the number of anticipated "events" would be high, and a study with adequate power could therefore be designed with a relatively small number of patients and would yield results in a relatively short period of time.

\section{Patterns of disease recurrence}

The $15 \%$ disease recurrence rate in the 367 SNB negative patients in our study is very similar to the $13 \%$ recurrence rate for SNB negative patients at three years' follow-up quoted by Gershenwald. ${ }^{4}$ The pattern of recurrence in these patients suggests that they should not be regarded as nuclear medicine, surgical, or pathological "failures," but rather that up to $15 \%$ of melanoma patients have a pattern of lymphatic or haematogenous tumour dissemination that bypasses the sentinel node or at the time of SNB have melanoma cells distal to the sentinel node and still in transit. Thus, although a negative SNB is an encouraging prognostic feature, patients will still need to be counselled that they may have a recurrence of their melanoma. In keeping with results from other groups, our data do not suggest that patients who have had SNB have a higher than expected incidence of in transit recurrence. ${ }^{13}$

\section{Conclusions}

Although knowledge of sentinel node status is an additional significant prognostic indicator, this knowledge does not change routine management of patients, as no widely accepted effective adjuvant treatment is available to offer to SNB positive patients after node dissection. The recently reported results of EORTC 19852 adjuvant interferon therapy for stage $2 \mathrm{~b}$ and 3 melanoma showed no overall survival benefit, ${ }^{14}$ and the early report of the benefit of interferon in the US study ECOG 1684 was not confirmed by the same group in ECOG $1690 . .^{15} 16$

We therefore suggest that until the full results of MSLT1 are published, sentinel node biopsy should not be a routine procedure for melanoma patients but should be used as a staging procedure in centres entering patients into adjuvant trials for patients with stage 3 melanoma. Knowledge of sentinel node status is necessary to stratify melanoma patients being entered into these trials and should be part of the protocol.

Contributors: SK, DS, AM, DB, TS, and BM did the sentinel node surgery; $\mathrm{CM}$ and RM did the pathological analysis; $\mathrm{CB}$ did statistical analyses; and RM followed up the patients and wrote the paper jointly with SK. RM is the guarantor.

Funding: None.

Competing interests: None declared.
Ethical approval: Glasgow West Ethical Committee (98/182/1).

1 Cabanas RM. An approach for the treatment of penile carcinoma. Cancer 1977;39:45666.

2 Morton DL, Wen DR, Wong JH, Economou JS, Cagle LA, Storm FK, et al. Technical details of intraoperative lymphatic mapping for early stage melanoma. Arch Surg 1992;127:392-9.

3 Balch CM, Soong SJ, Bartolucci AA, Urist, MM, Karakousis CP, Smith TJ, et al. Efficacy of an elective regional lymph node dissection of 1 to $4 \mathrm{~mm}$ thick melanomas for patients 60 years of age and younger. Ann Surg 1996;224:255-66.

4 Gershenwald JE, Thompson W, Mansfield PF, Lee JE, Colome MI, Tseng CH, et al. Multi-institutional melanoma lymphatic mapping experience: the prognostic value of sentinel lymph node status in 612 stage I or II melanoma patients. J Clin Oncol sentinel lymph
1999;17:976.

5 Doting MHE, Hoekstra HJ, Plukker JTM, Piers DA, Jager PL, Tiebosch ATMG, et al. Is Doting MHE, Hoekstra HJ, Plukker JTM, Piers DA, Jager PL, Tiebosch ATMG, et al. Is
sentinel node biopsy beneficial in melanoma patients? A report on 200 patients with cutaneous melanoma. Eur J Surg Oncol 2002;28:673-8

6 Morton DL, Hoon DSB, Cochran AJ, Turner RR, Essner R, Takeuchi H, et al. Lymphatic mapping and sentinel lymphadenectomy for early-stage melanoma: therapeutic utility and implications of nodal microanatomy and molecular staging for improving the accuracy of detection of nodal micrometastases. Ann Surg 2003;238:538-50.

7 Veronesi U, Paganelli G, Viale G, Galimberti V, Luini A, Zurrida S, et al. Sentinel lymph node biopsy and axillary dissection in breast cancer: results in a large series.J Natl Cancer Inst 1999;91:368-73.

8 Krag DN, Weaver DL, Alex JC, Fairbank JT. Surgical resection and radiolocalization of the sentinel node in breast cancer using gamma probe. Surg Oncol 1993;2:335-40.

9 Giuliano AE, Kirgan DM, Guenther JM, Morton DL. Lymphatic mapping and sentinel Giuliano AE, Kirgan DM, Guenther JM, Morton DL. Lymphatic m
lymphadenectomy for breast cancer. Ann Surg 1994;220:391-401.

10 Bergkvist L, Frisell J, Swedish Breast Cancer Group, Swedish Society of Breast Surgeons. Multicentre validation study of sentinel node biopsy for staging in breast cancer. BrJ Surg 2005;92:1221-4.

11 Fleissig A, Fallowfield LJ, Langridge CI, Johnson L, Newcombe RG, Dixon JM, et al Postoperative arm morbidity and quality of life: results of the ALMANAC randomised sentinel node biopsy with standard axillary treatment in the management of patients with early breast cancer. Breast Cancer Res Treat 2005;15:1-15.

12 Morton DL, Thompson JF, Cochran AJ, Essner R, Elashoff R. Interim results of the multicenter selective lymphadenectomy trial (MSLT-I) in clinical stage I melanoma. $J$ multicenter selective lymphadenectomy

13 Kretschmer L, Beckmann I, Thoms KM, Haenssle H, Bertsch HP, Neumann CH. Sentinel lymphadenectomy does not increase the risk of loco-regional cutaneous metastases of malignant melanoma. Eur J Cancer 2005;41:531-8.

14 Eggermont AM, Suciu S, MacKie R, Ruka W, Testori A, Kruit W, et al. Post-surgery adjuvant therapy with intermediate doses of interferon alpha 2 beta versus observation in patients with stage 11b/111 melanoma (EORTC 19852): randomised controlled trial. Lancet 2005;366:1189-96.

15 Kirkwood JM, Strawderman MH, Ernstoff MS, Smith TJ, Borden EC, Blum RH. Interferon alpha $2 \mathrm{~b}$ adjuvant therapy of high risk resected cutaneous melanoma: the Eastern Cooperative Oncology Group trial EST 1684. J Clin Oncol 1996;14:7-17.

16 Kirkwood JM, Ibrahim JG, Sondak VK, Richards J, Flaherty LE, Ernstoff MS. High and low dose interferon alpha 2 beta in high risk melanoma: first analysis of intergroup trial E1690/S9111/C9190.J Clin Oncol 2000;18:2444-58.

(Accepted 24 March 2006)

doi 10.1136/bmj.38849.680509.AE

\section{What is already known on this topic}

Before 1990, common practice in Europe was to widely excise the primary melanoma and then observe the patient, delaying lymph node surgery until nodes were palpable

North American practice was to offer elective node dissection of the appropriate draining nodal basin to most patients with primary melanomas thicker than $1 \mathrm{~mm}$

Many European centres are introducing sentinel node biopsy as a routine procedure despite the lack of evidence that this and completion lymphadenectomy extend overall survival

\section{What this study adds}

This prospective observational study shows that sentinel node status is a significant prognostic factor and is independent of both tumour thickness and ulceration

Until data from randomised trials are available, sentinel node biopsy status should be considered in patients entering adjuvant trials but should not become a routine standard of care 
Hairmyres Hospital, Lanarkshire G75 8RG

Stephen Kettlewell consultant vascular surgeon

Royal Alexandra Hospital, Paisley PA2 9PN

Colin Moyes consultant pathologist

Greater Glasgow NHS Board, Glasgow G3 8YZ

\section{Amendment}

This is version 2 of the paper. Deaths from other causes have been added to the abstract, and BM's place of work has been amended.
Caroline Bray statistician

Canniesburn Plastic Surgery Unit, Glasgow Royal Infirmary, Glasgow G4 OSF

David Soutar consultant plastic surgeon

Taimur Shoaib specialist registrar in plastic surgery

General Surgery Unit, Glasgow Royal Infirmary

Barun Majumder specialist surgical registrar

Gartnavel General Hospital, Glasgow G12 0YN

Alan MacKay consultant vascular surgeon

Dominique Byrne consultant vascular surgeon

Department of Public Health and Health Policy, University of Glasgow, Glasgow G12 8RZ

Rona MacKie senior research fellow

Correspondence to: R MacKie R.M.Mackie@clinmed.gla.ac.uk 